\title{
La France dans l'œuvre de Magali Michelet
}

Sathya RAO, Université de l'Alberta

\section{1. « Canadienne et doublement française »}

C'est en 1905 que les Michelet s'embarquent de France à bord du Dominion à destination de Winnipeg au Manitoba. À cette époque, la province attire une forte immigration française notamment en raison des campagnes de recrutement de certains pères oblats particulièrement actifs en France et en Belgique (Painchaud 32). Toutefois, c'est en Alberta dans un homestead non loin de Legal que le couple Michelet et leurs trois enfants élisent domicile ${ }^{1}$. Le ménage était très probablement de condition socio-économique modeste. François, le chef de famille, avait exercé de nombreux emplois en France, dont ceux de relieur, de policier, de marchand de porcelaine et de marchand forain (Rao, «Coin féminin », 132), mais c'est en tant qu'agriculteur qu'il figure dans la liste des passagers du Dominion ${ }^{2}$. Quant à son épouse, elle est sans profession au moment de son arrivée au Canada. Par ailleurs, nous savons que François Michelet était proche des milieux catholiques nantais et voyait d'un mauvais œil l'avènement d'une république laïque (Rao 133). Fervents catholiques en quête d'une vie meilleure, les Michelet avaient à quelques nuances près le profil que recherchaient les recruteurs oblats ${ }^{3}$.

Fille aînée de François et d'Hélène Michelet, Magali - de son vrai nom Marie Louise est âgée d'un peu plus de vingt ans lorsqu'elle débarque au Canada. Sur la liste des passagers du Dominion, elle est enregistrée comme «travailleuse domestique ». Cela dit, Michelet ${ }^{4}$ semble posséder un certain niveau d'éducation comme en atteste une lettre datée de 1925 dans laquelle elle affirme avoir obtenu « une première partie de "bachot" [...]» («Vie de l'action française », 387). Un an à peine après son installation à Legal, elle prend la charge de l'hebdomadaire francophone Le courrier de l'ouest avec l'aide de son frère Charles Alexandre. Pendant près d'une dizaine d'années, Michelet tiendra contre vents et marais la barre de la rubrique « Le coin féminin ». Parallèlement à sa tâche de chroniqueuse, elle entame une brillante carrière de dramaturge remportant coup sur coup le concours dramatique de l'Alliance artistique de Montréal en 1917, puis celui de l'Action française en 1921 avec les pièces «Jean Audrain » et Contre le flot. Sa troisième pièce «L'empreinte » soumis au concours d'art dramatique de 1924 connaît toutefois un succès plus mitigé en raison de son manque de caractère patriotique (« Notre 
concours », 63-64) et ne sera pas publié malgré les nombreuses missives de son auteure à l'abbé Groulx. Chroniqueuse de talent, Michelet voit certains de ses textes repris dans de prestigieuses revues littéraires de l'Est dont Le journal de Françoise de Robertine Barry que Michelet compte parmi ses amies et correspondantes (Rao, «Coin féminin », 145). Alors qu'elle est de retour en France au début des années 1920, Michelet est déjà une dramaturge reconnue au Canada français. Contre le flot a fait l'objet de nombreuses représentations qui ont donné à plusieurs recensions dans la presse nationale (Roy 292-293). En 1922, année de la publication de Contre le flot, paraît également la comédie en un acte Marraine de guerre publiée aux éditions McMillan French series. On peut supposer que c'est durant son séjour à Washington, où elle a suivi son frère récemment embauché comme interprète à l'Union Panaméricaine, que Michelet compose cette courte pièce. Destiné à un public d'apprenants du français, Marraine de guerre met en scène la rencontre entre Suzanne de Brémont et le capitaine D. Gibbs avec qui cette dernière a entretenu une correspondance durant la Grande Guerre. Compte tenu de son thème même et de l'importance qu'elle confère à l'échange épistolaire, cette pièce préfigure Comme jadis publié en 1925. Issue d'un milieu modeste, Michelet a connu au Canada un destin littéraire dont elle n'aurait pu rêver en France où elle est demeurée, même après son retour au milieu des années 1920, une illustre inconnue 5 . L'exil forcé au Canada aura ainsi été pour elle l'occasion d'un changement de statut inespéré.

Michelet fait partie de ces écrivains d'origine française qui, comme M. Constantin-Weyer et G. Bugnet, ont accordé une place centrale à l'Ouest canadien dans leur œuvre littéraire. Moins connue que ses compatriotes, Michelet a pourtant compté parmi les grands auteurs canadiens français de son temps. À ce titre, elle ne peut échapper à la comparaison avec l'auteur d'Un homme se penche sur son passé $e^{6}$ dont les romans ont donné de l'Ouest canadien une image pour le moins controversée. Ce parallèle fréquemment évoqué par les critiques tourne généralement à l'avantage de Michelet dont la durée du séjour canadien garantirait l'authenticité du récit :

Seul un écrivain ayant longtemps vécu dans l'Ouest pouvait en décrire avec une telle précision les divers aspects, et analyser avec tant de justesse les sentiments des pionniers canadiens-français. M. Constantin-Weyer dans son Manitoba, où d'ailleurs éclate un grand talent, s'était naguère donné la même tâche. Il a, selon nous, gâté son œuvre par de graves manques de goût et pas mal de persiflage. Ici rien de tel. On dirait même que Magali a entrepris de nous montrer qu'il eût été facile à M. Constantin-Weyer de ne pas tomber dans les défauts que j'ai signalés tantôt. Elle aussi décrit la vie des fermes de l'Ouest, elle aussi rencontre une famille canadienne-française, elle aussi termine son récit à la mobilisation. Mais 
nulle part, elle ne verse dans la médecine vétérinaire, dans le récit grotesque ou dans le discours suffisant du patriote chauvin. (Deligny 55)

Et qui de mieux placé pour attester de cette authenticité qu'un autre expatrié français ? C'est bien ce que fait Donatien Frémont dans son ouvrage Sur le ranch de Constantin-Weyer. Tandis qu'il reproche à l'auteur d'Un homme se penche sur son passé d'avoir travesti la réalité de l'Ouest canadien, il brosse un portrait élogieux de l'œuvre de cette «Française qui a vécu longtemps dans le nord de l'Alberta » :

Dans Comme jadis, elle a décrit avec étonnante précision et un charme exquis les débuts d'un petit centre de colonisation auxquels elle avait elle-même assisté. La répercussion causée dans ce milieu bien français par le déclenchement de la Grande Guerre et les longues angoisses qui suivirent ont inspiré à l'auteur des pages émouvantes et d'une incontestable originalité. Le roman de Mlle Michelet, peu connu du public, renseigne mieux sur la vie des colons dans l'Ouest canadien que tous les livres de M. Constantin-Weyer (66-67).

L'authenticité dont Frémont se fait le porte-parole va de pair avec une esthétique de la sincérité qui commande au romancier de n'écrire rien d'autre que les faits. C'est bien à ce titre que l'historien met en question l'œuvre de Constantin-Weyer :

Jusqu'où va, au fond, chez M. Constantin-Weyer, la sincérité du récit? À quel point fut-il mêlé aux aventures dont il est le narrateur et le héros? De quelle valeur documentaire est sa peinture des métis français d'aujourd'hui et de leurs ancêtres du siècle dernier? Quel cas fait de la vérité historique l'écrivain qui met en scène des personnages réels et prétend reconstituer les épisodes les plus dramatiques de l'histoire de l'Ouest canadien? (9)

Comme le remarque à juste titre Robert Viau, « un tel réductionnisme transforme l'œuvre fictive en un témoignage sociohistorique, nie l'existence d'un espace textuel romanesque différent de l'espace strictement référentiel » (59).

Devant un lectorat canadien-français qui craint qu'on donne de lui une image plus vraie que nature et reproche aux Français leur anticléricalisme, Michelet doit faire la preuve de sa bonne foi. Son adhésion pleine et entière aux valeurs canadiennes-françaises dont se fait l'écho la pièce Contre le flot - qui a reçu les honneurs du journal de l'Action française - contribue temporairement à faire oublier ses origines hexagonales et la propulse au panthéon de la littérature canadienne-française aux côtés d'un autre Français :

Maria Chapdelaine, un chef-d'œuvre renferme plusieurs faux tons dont l'oreille canadienne souffre, des dissonances fâcheuses. Rien de tel dans Contre le flot - et veuillez croire que je ne compare pas la statuette à la statue, et mets l'une à la place d'honneur, l'autre dans un coin. Mais la pièce représentée hier soir trahit par 
quelques nuances son origine. L'auteur est un observateur qui voit juste, un psychologue qui a saisi exactement les mouvements des âmes.

C'est bien la vie canadienne, c'est bien l'opposition entre les renégats et les représentants du patriotisme intégral. L'un des nôtres n'aurait pas dit autre chose ; mais, à certains endroits, il l'aurait dit autrement («Première de Contre le flot », 27)

Le retour de Michelet en France au moment où sa pièce connaît un succès national lève définitivement le doute sur son appartenance. D'après le témoignage de sa nièce, il semble qu'elle ait continué à entretenir un intérêt particulier pour le Canada et son actualité (Rao «entretien »). Dans une lettre datée de 1955 adressée à l'abbé Groulx, elle évoque même la rédaction d'un roman qui serait le « fruit de mes [ses] années canadiennes » et nourrit le projet de faire des émissions de radio sur les grandes figures féminines du Canada francophone. Toutefois, à notre connaissance, aucun de ces projets ne semblent s'être concrétisés. Aux yeux de nombre de Canadiens français, elle restera à jamais cette «noble Française » qui aura su témoigner dans ses œuvres d'une sympathie réelle à l'endroit de la cause canadienne-française (Michelet, «Vie de l'action française », 263).

Suite à une erreur commise sur la durée de son séjour au Canada dans un compte rendu tiré de l'Action française, l'auteure fait une mise au point particulièrement éclairante sur sa canadianité :

Parlant du lointain pays, où m'attachent tant de souvenirs inoubliables, il m'arrive souvent de dire «chez nous ». Et il me semble avoir quelques droits à cette expression. Je n'aurai pas l'orgueil de croire que j'aie contribué à la «construction» de ce foyer d'ardente pensée et d'action française élevée en Alberta-Nord, mais invinciblement je songe avec un sentiment de fierté attendrie au «Coin féminin » du Courrier de l'Ouest et au champ de contours irréguliers... Tout paraissait devoir me lier à jamais au cher coin du pays adoptif ; la vie avec ses remous et an décidé autrement. Je suis partie, mais au Vieux Pays j'ai gardé, chaude, au cœur, la devise «Je me souviens ». D'être canadienne aussi, il me semble être doublement française (387-388).

L'erreur dont Michelet est malencontreusement l'objet et la mise au point qui en découle, témoignent bien des préjugés dont Michelet est victime de la part de ses frères d'armes de l'Action française et de l'institution littéraire canadienne-française dans son ensemble. Ainsi dans la notice que lui consacre Georges Bellerive est-il précisé à toutes fins utiles que l'auteure bien que d'origine française a reçu «sa formation littéraire au Canada » (132). Quant à l'entrée concernant Comme jadis dans le Dictionnaire des auvres littéraires du Québec rédigée quelque cinquante ans plus tard, elle indique que le roman demeure «l'œuvre d'un écrivain étranger» 
(Gaulin 263) à la solde de l'idéologie clérico-nationaliste défendue par l'abbé Groulx et ses partisans. Pour Max Roy, auteur de l'entrée sur «Contre le flot » dans le même dictionnaire, l'«écriture un peu maniérée » de Michelet «trahit ses origines françaises » (292). Assimilée au mouvement l'Action française et à ses dérives, l'auteure se trouve désormais reléguée aux oubliettes de l'histoire littéraire canadienne. Aujourd'hui il n'y a guère plus que Le dictionnaire des artistes et auteurs francophones de l'Ouest qui lui consacre une entrée digne de ce nom. Dans le cadre de cette étude, notre propos ne sera pas tant de la réhabiliter que de jeter un regard plus contemporain sur son œuvre. Plus exactement, nous nous intéresserons à la façon dont Contre le flot, Comme jadis et «Le coin féminin » mettent en scène le rapport entre la France et le Canada ${ }^{7}$. Nous montrerons que l'œuvre de Michelet est moderne surtout en raison de sa capacité à produire une image de l'Ouest (et plus largement du Canada) qui s'écarte des schèmes narratifs convenus du roman d'aventures ou de la chronique de pionnier. Quant au « Coin féminin », il se situe au carrefour de plusieurs francophonies, ce qui en fait un espace inédit de dialogue.

\section{L'œuvre de Magali Michelet : à mi-chemin entre le Canada et la France}

a) Contre le flot et le statut symbolique de la France

Contre le flot suit de quelques années «Jean Audrain » qui avait été récompensée par l'Alliance artistique de Montréal en 1917. Cette pièce de jeunesse dont il ne reste malheureusement aucune trace écrite à notre connaissance, s'attachait à explorer le dilemme posé par la Grande Guerre pour nombre d'expatriés français déchirés entre leur devoir patriotique et leur désir de s'établir au Canada. Pièce lauréate du premier concours d'art dramatique organisé en 1921 par la revue de l'Action française sur le thème de l'anglomanie, Contre le flot abandonne toute référence explicite à la France pour faire la promotion des valeurs canadiennes-françaises (sentiment religieux, exhalation patriotique, défense du fait français). Il n'y est plus question des difficultés de l'exil, mais plutôt du combat pour la survivance du fait français. Selon l'intitulé du concours lancé par la revue, il fallait entendre par anglomanie :

Non seulement la tendance déplorable qui consiste à renier sa langue au profit de celle du conquérant, mais un état d'âme qui se manifeste, d'une façon générale, par le mépris de sa race et l'admiration exclusive des choses anglaises. L'anglomanie qu'il paraît urgent d'atteindre par le ridicule et la satire, c'est proprement le Canadien français follement entiché de la supériorité anglo-saxonne 
et qui, par vanité ou par calcul, pousse systématiquement les siens vers l'abdication nationale et la fusion des races (Trofimenkoff 178).

Dans son ouvrage consacré à l'Action française, Susan Mann Trofimenkoff rapporte le mécontentement de l'écrivain Napoléon Lafortune qui, dans une lettre adressée à l'Abbé Groulx, déplore que ce soit l'œuvre d'une femme et qui plus est d'une Française qui ait été récompensée (43). Pour autant, la France ne disparait complètement de la pièce ; elle y fait l'objet de quelques références éparses. En premier lieu, le docteur André Lamarche, protagoniste principal de Contre le flot, revient justement d'un séjour à Paris au cours duquel il a conduit des recherches sur le cancer aux côtés de son ancien professeur, le Dr Fortin (20). D'un point de vue diégétique, ce séjour marque la rupture avec Corinne Cantin avec qui André devait se marier et qui, au mépris de l'«appel de sa race », lui préférera un riche homme d'affaires anglophone. Pour le Canadien français qu'est le docteur Lamarche, la France est bien plus qu'un lieu de formation intellectuelle :

Là-bas [à Paris], j'ai appris, oui, en vérité, j'ai appris beaucoup sous la direction de professeurs admirables... Je reviens avec l'espoir de pouvoir achever les travaux du Dr Fortin... Je vous confie tout bas ; j'ai appris aussi, ce qu'on ne m'avait pas assez enseigné ici : la fierté que nous devons à notre race... Non pas la fierté dont nous enivre la phrase claironnante d'un discours de St-Jean-Baptiste - encore que la respecte celle-là, parce qu'elle nous conserve, d'années en années, l'âme chaude du peuple -, mais la fierté de vouloir... la fierté d'égaler... la fierté de s'être retrouvé en s'abreuvant à la source du génie de la race... Je sais mieux ce que nous devons vouloir (26-27).

Elle est la source originaire dont il convient de s’inspirer ; le génie français a ses héros religieux, scientifiques et militaires - dont les noms sont égrenés au fil de la pièce : Vincent de Paul (27), Pasteur (27) Joffre (43), Foch (43). La deuxième référence à la France concerne la Grande Guerre à laquelle a pris part le docteur Lamarche. Centrale dans «Jean Audrain », la Première Guerre mondiale n'est évoquée qu'à trois reprises dans Contre le flot, deux fois brièvement par le docteur lui-même $(49 ; 72)$ et une fois par Marie-Blanche (43), mais de façon plus substantielle, cette fois. Son propos est surtout de dénoncer l'inégalité de traitement dont font l'objet les soldats canadiens-français par rapport aux canadiens-anglais. Enfin, dans un tout autre contexte, on retrouve une mention à la France de la part de Mrs Miller, qui se vante de parler le «français de Paris » (9). Marcelle, son interlocutrice, voit dans cette allusion la marque d'un snobisme, voire d'un dédain à l'endroit du français parlé par les Canadiens français. Contrairement à «Jean Audrain », Contre le flot s'ancre fermement en sol canadien, d'abord à 
Westmount où se déroule le premier acte, puis dans l'Ouest qui sert de cadre aux deux derniers actes. La France n'y a plus qu'une présence lointaine et essentiellement symbolique comme source d'inspiration ou modèle parfois invoqué à mauvais escient à l'image de la francomanie de Mrs Miller. Il semble donc que le combat se soit déplacé de la France vers le Canada français qui constitue désormais un nouveau champ de bataille. Ce combat dont la pièce évoque les prolongements politiques, économiques et sociaux, est celui pour la préservation du fait français. Héros canadien-français par excellence, le docteur Lamarche devra lutter contre la passion qu'il éprouve pour Corinne l'anglomane tout en veillant à ne pas compromettre son intégrité dans des jeux politiques destinés à financer sa recherche. C'est finalement de la communauté francophone que viendra le salut. Le Consul de France se joindra à cet « effort de guerre » en apportant à son tour son soutien financier aux recherches du docteur Lamarche. Fermement enraciné dans le terroir et les valeurs canadiennes-françaises, Contre le flot donne à la France un rôle qui, pour être important d'un point de vue symbolique, n'en est pas moins secondaire en ce qui a trait à l'intrigue. Nous verrons que la France occupe une tout autre place dans Comme jadis.

\section{b) Comme jadis et le télescopage des chronotopes}

Rédigé en France très certainement dans les années qui suivent son départ du Canada ${ }^{8}$, Comme jadis a été publié à Montréal dans la collection de la bibliothèque de l'Action française fondée en 1919. Placée sous le haut patronage de Napoléon Lafortune, cette collection diffusait essentiellement la propagande littéraire de l'Action française ainsi que quelques ouvrages de nationalistes français comme Léon Daudet, Charles Maurras et Jacques Bainville (Troffimenkoff 42). Figurant parmi les premières publications de la maison d'édition, Comme jadis est remarquable en raison de sa forme épistolaire qui abolit les distances tout en modifiant la perception l'espace, qui devient fonction du temps, c'est-à-dire des départs de malle, des retards de courrier, de l'attente angoissante ou encore du temps physique de l'écriture assujetti aux contraintes du quotidien. Au fil de la correspondance entre Gérard de Noulaine et Herminie Lavernes (également désignée dans le roman par le diminutif de «Minnie »), la distance géographique entre la France et le Canada semble se dématérialiser à mesure que se trouve convoquée leur histoire commune. Ainsi la correspondance entre Gérard et Herminie ${ }^{9}$ d'un bord à l'autre de l'Atlantique prolonge-t-elle celle ayant eu cours entre leurs aïeux cent cinquante ans plus tôt et dont Gérard a publié une partie sous forme de roman. Comme jadis devient de la sorte le lieu d'improbables retrouvailles où convergent passé et présent. À cet égard, l'illusion 
d'optique que produit le cliché photographique représentant Herminie posant à la manière de son aïeule peinte sur une étampe que Gérard lui a fait parvenir est emblématique. Miraculeuses, ces retrouvailles n'en demeurent pas moins marquées du sceau tragique du destin: Gérard ne sait pas encore qu'il mourra au front comme son ancêtre dont il porte le prénom. Quant à Herminie, elle est condamnée à connaître le même sort que son arrière cousine : perdre celui qu'elle aime (269) et vouer son amour à Dieu. Cela dit, l'attention portée au présent des événements narrés si caractéristique de l'effet de «myopie » (Rousset) des romans épistolaires fait perdre de vue la tragédie à venir et redonne pour ainsi dire espoir. En définitive, la mécanique épistolaire produit un télescopage des chronotopes : le passé de la bataille pour les plaines d'Abraham de 1759 au Québec se surimpose avec le présent de la Première Guerre mondiale en France. Tandis que le conflit se fait imminent, Gérard et Herminie prennent conscience que le destin les rattrape :

À plus de cinquante ans de distance, la Volonté divine a voulu qu'une nouvelle Herminie de Lavernes, un nouveau Gérard de Noulaine se trouvent placés dans un $^{10}$ situation identique à celle du Chevalier et de la noble demoiselle. Comme jadis ils se sont aimés et soutenus, nous nous soutiendrons et nous nous aimerons. Et même si notre roman s'achevait comme le leur, Minnie, il faudrait rendre grâce à Dieu qui a rapproché un instant les deux rameaux du même arbre (257).

Comme son titre le laisse penser, Comme jadis apparaît comme une tentative de transgresser l'espace par le temps, qu'il s'agisse du temps historique objectif ou bien du temps intime de la correspondance. Dématérialisée d'un point de vue référentiel, la distance l'est également sur le plan pragmatique de l'écriture. En effet, le choix de la forme épistolaire instaure une double proximité entre le lecteur et le narrateur qui partagent de fait une commune intimité, mais aussi entre le narrateur et son personnage, lequel prend pour ainsi dire la parole. Dressant un parallèle intéressant entre roman par lettres et théâtre Jean Rousset note à cet égard :

Dans le roman par lettres - comme au théâtre -, les personnages disent leur vie en même temps qu'ils la vivent ; le lecteur est rendu contemporain de l'action, il la vit dans le moment même où elle est vécue et écrite par le personnage ; car celuici, à la différence cette fois du héros de théâtre, écrit ce qu'il est en train de vivre et vit ce qu'il écrit ; plus complètement qu'au théâtre, il se substitue à l'auteur et l'évince, puisqu'il est lui-même l'écrivain ; personne ne parle ni ne pense à sa place, c'est lui qui tient la plume (67-68).

En somme, le télescopage de l'espace-temps combiné à l'immédiateté de la performance d'écriture achève d'abolir les distances. En ce sens, Comme jadis opère un déplacement topique des représentations littéraires traditionnelles de l'Ouest. L'espace qui y est convoqué n'a pas le relief qui lui confère un Constantin-Weyer dans ses romans (Tessier 119); il n'est pas la scène 
grandiose d'une action dont il est lui-même partie prenante en s'opposant de toute son immensité à la volonté de maîtrise des hommes (Viau 72). Dans Comme jadis, l'espace est moins prétexte à une progression linéaire (par exemple, sous les espèces d'une lutte contre la nature) qu'à un partage dont la correspondance épistolaire constitue le véhicule. Si les histoires de pionniers que rapporte Herminie n’ont certes pas le souffle épique de celles narrées par Constantin-Weyer, elles n'en dégagent pas moins une impression de sincérité à laquelle Frémont n'aura pas été insensible. Toutefois, cette sincérité ne procède pas d'un travail de reconstruction historique comme c'est le cas de plusieurs chroniques de pionniers (Durieux, Maturié) comme nous le montrerons dans la suite de notre article. Elle n'a pas non plus vocation à titiller l'imaginaire du lecteur pour qu'il se prenne au jeu de ses propres illusions nourries par les romans d'aventures. Dans le compte rendu de Comme jadis qu'il fait dans Le devoir, Edmond Léo (pseudonyme d'Armand Chossegros) insiste justement sur le caractère « vivant » du roman qui « raconte la vie des pionniers et ne les fait pas agir dans une action dramatique » (1). «Incontestablement original », le roman de Michelet l'est non pas en raison de sa véracité comme l'écrivait Frémont, mais dans la mesure où il donne à lire une expérience à la fois immédiate et intimiste de l'Ouest. En marge des grands récits triomphants de la conquête, l'Ouest de Michelet est vécu dans l'intimité quotidienne du foyer qu'Herminie partage avec son père, Nanine, ainsi qu'un couple de Français, les Mourier. D’apparence anodine, cette intimité possède néanmoins le pouvoir de démystifier ces grands récits, qu'il s'agisse des romans d'aventures...

Vous n'imaginez pas que je chasse le bœuf musqué ou que je fume le calumet de la paix au wigwam voisin ? Qui sait? Vous craignez peut-être pour mon scalp...! Hélas! Dans ma maison perdue en pays neuf - je devrais dire, en pays sauvage je ne cours guère plus de dangers que n'importe quelle jeune fille française habitant au cœur de la province la plus tranquille (69).

Ou bien de la propagande officielle encourageant la colonisation au Canada ${ }^{11}$ :

On a écrit, en ces dernières années, des pages et des pages sur le développement du Canada. Peut-être, par hasard, en avez-vous lu quelques-unes ? L'Ouest, particulièrement, a excité la curiosité du Vieux-Monde. On a vanté ses richesses minières et agricoles, on a fait miroiter ses «opportunités »; ce qu'on ne dira jamais assez c'est la vaillance, la fermeté dans la confiance dont durent faire preuve les premiers occupants (27).

Si le roman épistolaire s'inscrit dans une longue tradition littéraire qui compte parmi ses plus dignes représentants Balzac et la Marquise de Sévigné, il procède en premier lieu d'une pratique ordinaire à laquelle des colons avaient fréquemment recours pour donner de leurs 
nouvelles à leurs proches. Dans sa forme même, le roman de Michelet consacre cette pratique à laquelle «Le coin féminin » avait donné ses lettres de noblesse vingt ans plus tôt. En fin de compte, Comme jadis investit l'Ouest d'une nouvelle forme littéraire qui se distingue à la fois du roman d'aventures et de la chronique de pionniers. Bien avant Aux pays des érables d'Amélie Constantin-Bompard ${ }^{12}$, l'œuvre de Michelet offre une perspective inédite - que l'on pourrait qualifier de féminine (plutôt que de féministe ${ }^{13}$ ) - sur l'Ouest en porte-à-faux des récits conquérants de pionniers. Quelque soixante ans après la publication du roman de Michelet, $D u$ vent, Gatine de Jacques Bertin remettra au goût du jour le roman épistolaire en utilisant la correspondance de colons français partis au Canada comme trame de fond. Cela dit, plutôt que de constituer le corps du roman à proprement parler, les lettres sont insérées dans le corps du texte pour faire l'objet de commentaires et de mises en contexte.

La forme épistolaire trouve à nouveau à s'arrimer à la réalité historique avec les lettres qu'envoie Gérard du front. Le référent extralinguistique est cette fois la lettre du poilu qui, tout comme celle du pionnier, confère à la fiction épistolaire un effet de réel, une actualité historique. Que ce soit à titre de chroniqueuse, de dramaturge ou bien de romancière, Michelet place la correspondance au cœur de son œuvre, rappelant par là même l'importance de cette pratique très ordinaire à l'époque. À cet égard, on ne manquera pas de souligner que depuis une dizaine d'années, un certain nombre de travaux dans le domaine de l'histoire et de l'ethnographie à l'instar de ceux regroupés dans le collectif Envoyer et recevoir. Lettres et correspondances dans les diasporas francophones tirent de l'examen des correspondances une connaissance renouvelée de l'expérience migratoire ${ }^{14}$. Roman épistolaire, Comme jadis renouvelle ainsi par sa forme même la représentation littéraire de l'Ouest.

Toutefois, cet effet de réel doit être nuancé dans la mesure où plusieurs lettres de Gérard et surtout d'Herminie se présentent sous la forme de longs récits intradiégétiques de nature descriptive qui rappellent à plus d'un égard les chroniques de pionniers. L'insertion de dialogues dans ces lettres (65) au mépris de toute vraisemblance tend à renforcer le caractère romanesque de Comme jadis au détriment de son aspect proprement épistolaire. En ce sens, le roman de Michelet se démarque d'Angéline de Montbrun plus proche du format épistolaire traditionnel à plusieurs voix. Toutefois, les rappels à la situation fictive d'énonciation clôturant les lettres ainsi que les multiples interpellations à l'adresse du destinataire participent bien de la forme épistolaire. À l'évidence, Herminie a à cœur de donner à son interlocuteur - et par extension, à 
ses lecteurs - une image de l'Ouest canadien qui pour être intime n'en est pas moins authentique. C'est probablement à ce souci d'authenticité que répond la présence de nombreux emprunts au français canadien et à l'anglais - en italique ou entre guillemets dans le texte - dont «sleigh» (37), «trail » (39), «cernages » (40), «cayuse » (44), «bogheis » (50) qui donnent aux lettres d'Herminie une couleur locale. De même, certains protagonistes du roman sont très probablement inspirés de personnages réels, c'est notamment le cas de la figure tutélaire du père Chassaing d'origine française dont l'engagement indéfectible en faveur de la colonisation rappelle celui de missionnaires oblats français comme Émile Graire et Louis-Pierre Gravel.

Contrairement aux romans de Constantin-Weyer sur l'Ouest canadien tous publiés en France, Comme jadis paraît à Montréal à l'instar de la première version de Maria Chapdelaine ${ }^{15}$. Si les œuvres de l'auteur de Manitoba ainsi que le roman de Louis Hémon sont avant tout destinées au public français (Boivin 7), qu'en est-il du texte de Michelet ? Si le choix du lieu de publication laisserait croire que le lectorat visé par l'auteur est canadien-français, la forme épistolaire du roman vient, elle, toutefois compliquer cette hypothèse. En effet, si l'on se place du point de vue d'Herminie, le destinataire n'est autre que Gérard de Noulaine dont on peut penser qu'il agit également à titre de représentant métonymique du lectorat français. À ce titre, il est intéressant de noter que le roman a connu une republication en France en 1933 dans la collection «Stella » du magazine féminin Petit écho de la mode ${ }^{16}$. Bien que parfois naïves, les réactions de Gérard n'en sont pas moins bienveillantes à l'image de l'enthousiasme enfantin qui le saisit lorsqu'il se découvre une cousine canadienne : «Alors c'est vrai ? J'ai une cousine dans ce grand et lointain Canada qui m'intrigue comme une énigme ? Votre chère lettre m'a restitué mon âme enfantine: je l'ai lue avec l'avidité qui me faisait dévorer en une heure - il y a longtemps - un livre de Gustave Aymard ${ }^{17}$. Ma cousine, vous avez narré le plus délicieux conte qui soit » (54). Cela dit, il n'est pas certain que le lectorat canadien-français partage ce genre d'enthousiasme. D'autant plus que ce lectorat conserve une certaine amertume à l'égard de ces frères et cousins qui ont «déserté » la vieille province ainsi que le rapporte Herminie prompte cependant à prendre la défense des francophones de l'Ouest :

Je suis fille de l'Ouest. Là-bas, en province de Québec, durant le temps de mes études, j'ai souvent senti qu'on professait un peu de mépris pour la fille des prairies. Aux yeux de nos frères de la vieille province, nous avons perdu l'intégrité de notre nationalité canadienne-française ; nous sommes partis, nous avons affaibli la cause par notre désertion... La cruauté de ce paradoxe est peut- 
être le plus puissant stimulant des groupements canadiens de l'Ouest. Comment peut-on douter des liens qui nous rattachent au Québec? (110).

Alors qu'elle circule dans un «char » pour se rendre chez Mme Lamarche, Minnie fait la rencontre d'une famille de Québécois dont elle précise que l'histoire « ressemble à celle de Lavernes, à celle de chaque paroisse » (213). Le poids de cette histoire commune ne peut que renforcer le lien privilégié entre Canadiens-français et Français, que l'éloignement géographique tend à plonger dans l'oubli. Dans le compte rendu du roman qu'il signe pour la revue de l'Action française, Louis Deligny loue justement la fibre nationaliste de Comme jadis : «Je ne sache pas d'endroit où son coup d'œil m'apparaisse plus juste que lorsqu'il entreprend de définir le patriotisme canadien (p. 174), la lutte canadienne (p. 197), et lorsqu'il met en garde le voyageur français contre des impressions superficielles et des jugements hâtifs » (56). Parfois coupable d' « impressions superficielles », la France qu'incarne Gérard de Noulaine n'en est pas moins très critique de son propre désintérêt à l'endroit de ses cousins d'Amérique :

Qu'avons-nous fait, nous, catholiques et français, pour établir de solides liens fraternels avec cette France d'Amérique ? Nous ne savons rien ou presque, de ses aspirations. Les quelques Français, moins ignorants que les autres de son attachement aux traditions, se perdent à vouloir expliquer un patriotisme fait d'ardente intransigeance nationaliste et de loyalisme à la Couronne d'Angleterre. Et, de là-bas, comment nous voit-on ? Nous juge-t-on d'après notre détestable politique ? Que faisons-nous pour nous faire connaître ? Parcourir ce jeune pays en voyage d'études serait intéressant... (169)

D’une teneur différente, les lettres d'Herminie et de Gérard reflètent la position énonciative de leurs auteurs. Essentiellement descriptives, les premières s'attachent à dépeindre la vie dans l'Ouest canadien ainsi que la situation des francophones qui y demeurent. À ce titre, on peut penser qu'elles répondent, avec les précautions qui s'imposent, à la curiosité du lectorat français et renforcent la sympathie du lectorat canadien-français à l'égard de ses compatriotes de l'Ouest. Quant aux lettres de Gérard, elles portent principalement sur ses tourments intérieurs on parlerait aujourd'hui de dépression - suite à sa rupture amoureuse avec Jacqueline Maurane. Leur ton est donc plus introspectif et psychologique. Cette opposition - entre extériorité et intériorité - sert de trame à un antagonisme plutôt convenu entre vieux continent et Nouveau Monde qu'Herminie évoque dans les termes suivants :

La vérité est que je ne me trouvais jamais assez ferme pour être l'amie que vous réclamiez. N'est-ce pas invraisemblable que, moi, la petite Canadienne, perdue dans la solitude du Nord, fruste et sans expérience du monde, je puisse être d'un 
secours appréciable à Gérard de Noulaine, le peintre déjà célèbre, habitué aux finesses d'esprit et aux expressions de ses compatriotes si cultivés ? (129)

À la jeunesse et à la vigueur d'Herminie pour qui la « vraie vie c'est la vie au plein air » (155) s'oppose l'immobilisme et la souffrance psychologique d'un Gérard, prostré dans sa solitude, qui se décrit dans ses lettres comme un «vieil homme» (57). Au fil de la correspondance, cet apparent clivage donnera matière à un véritable échange duquel chacun des interlocuteurs sortira enrichi. Pour Gérard, les lettres d'Herminie revêtent une fonction thérapeutique et énergisante : «Minnie, toute ma volonté s'appliquera à vous comprendre. Vous me direz ce que vous exigez de moi. Vous me montrerez le chemin. Déjà le frémissement de votre énergie me gagne en ondes douces et impératives. Laissez s'achever ma convalescence ; je veux guérir (75). Les lettres d'Herminie sont chargées de toute l'énergie brute que requiert sa condition de pionnière. C'est cette énergie qui poussera Gérard à sortir de sa léthargie pour prononcer une conférence sur la Nouvelle-France et même à vouloir y entreprendre un « voyage d'études » à la manière de Tocqueville. Dans une lettre envoyée du front, Gérard va même jusqu'à dresser un parallèle entre la «vaillance » d'Herminie qui œuvre à sa tâche et le courage dont doit faire preuve le soldat. Dans un cas comme dans l'autre, il importe «d'agir, Ne pas se replier sur soi » (268). En définitive, l'Amérique française a quelque chose de profondément inspirant et même de rassérénant pour ces Français qui comme Jacqueline Maurane ${ }^{18}$ sont obnubilés par «le succès à tout prix » (97). Il faudrait bien se garder de confondre la morale du pionnier avec celle du «bon sauvage » qui n'est guidée que par ses propres instincts. Tel que le conçoit Michelet, le pionnier est avant tout l'artisan d'un retour à la terre et au catholicisme qu'elle reproche à la France industrielle et républicaine d'avoir abandonné. Malgré la distance qui les sépare, Gérard et Herminie partagent ces idéaux. En ce sens, Comme jadis, malgré sa forme singulière, adhère pleinement à l'idéologie du terroir promue par l'Action française de l'Abbé Groulx dont on retrouve des échos dans l'Action française de Charles Maurras (Trofimenkoff 20) sans qui ne faille toutefois les assimiler l'une à l'autre (Dionne 19). Profondément inspirée par cette idéologie, l'œuvre d'Herminie (et de Michelet) possède un caractère exemplaire la démarquant des chroniques traditionnelles de pionniers qui sacrifient toute visée politique au profit du réalisme de la reconstruction historique (Durieu, Maturié) ou bien orientent leur récit en fonction de la propagande officielle (Forestier). Que tire Herminie, pour sa part, de son échange avec Gérard ? Outre le fait de reprendre contact avec son histoire 
familiale, elle trouve enfin l'occasion d'exercer son empathie naturelle, d'accomplir sa vocation de femme. Au fil de la correspondance, cette empathie se transforme en écoute, en amitié fraternelle puis en amour, pour finir par se mettre au service de Dieu suite au décès de son bienaimé.

L'originalité de Comme jadis est de produire une situation de coénonciation ${ }^{19}$ qui rend possible un point de vue que l'on pourrait qualifier de stéréoscopique sur le Canada et la France. Un tel point de vue permet à la fois de corriger les excès de part et d'autre - qu'il s'agisse de la vision déformée de la réalité canadienne que proposent les romans d'aventures ou bien des travers « romanesques » (163) d'Herminie - tout en mettant en lumière ce qu'il y a de commun entre ces deux pays issus après tout de la même «souche » comme il est dit dans le roman. Cette vision stéréoscopique inspire à Michelet une galerie de portraits diversifiés quoique familiers aux yeux des lectorats canadiens et français, ainsi que le note Deligny (56) : le couple de Français expatrié dans l'Ouest ; la famille d'immigrants québécois ; le père oblat d'origine française ; Henri Maignan, le fils d'industriel guidé par des idéaux chrétiens ; Marthe Leray la «sainte laïque »; les ouvriers nantais à la recherche d'une vie meilleure au Canada ${ }^{20}$, etc. Ancrée dans le passé d'une histoire commune, cette coénonciation ouvre la perspective d'un avenir fondé sur des valeurs partagées - le nationalisme, le patriotisme, l'agriculturisme - sur lesquelles l'Action française de l'abbé Groulx et celle de Charles Maurras ne manqueraient pas de s'accorder ${ }^{21}$. Cet avenir paraîtra sans nul doute rétrograde à la majorité des Français qui ont épousé les valeurs révolutionnaires de la République laïque de Jules Ferry. C'est par le sacrifice certes tragique, mais assumé au nom de la protection de la protection de la patrie ${ }^{22}$, que se conclut Comme jadis et que l'histoire finit par se répéter. Contrairement aux chroniques de pionniers comme celles de Marcel Durieux, de Pierre Maturié et de Georges Forestier qui mettent en scène un récit linéaire de la conquête de l'Ouest qu'interrompt brutalement la Grande Guerre, Comme jadis a le mérite de construire une temporalité plus complexe où passé et présent se répondent. Ainsi la conquête de l'Ouest se surimpose-t-elle à la fois à l'histoire de la fondation de la Nouvelle-France et à l'engagement dans la Grande Guerre en ce qu'il y est chaque fois question de vaillance (27; 266).

On ne manquera pas de conclure cette partie en signalant que Comme jadis contient quelques références intertextuelles à Contre le flot. On y retrouve en effet le protagoniste principal de la pièce, le docteur André Lamarche (217), ami de longue date des Lavernes. De 
même, l'auteur égratigne au passage ces « snobinettes » qui, à l'instar de Mlle Cantin, cultivent l'anglomanie à tout prix :

- Que venez-vous étudier, Monsieur ? Notre constance dans les traditions françaises ? Nous nous américanisons et c'est tant mieux ! Parler français est habitant... Le mariage mixte est très chic... C'est vrai, les enfants ne sont pas toujours élevés dans la religion de la partie catholique, mais notre clergé est bien exigeant... Je vous assure, tout est très bien ainsi ; laissez-nous courir nos chances avec les « États »!... (197).

Enfin, Comme jadis contient des similarités troublantes avec une des chroniques du «Coin féminin » intitulée « Roman d'aïeule » publiée dans Le courrier de l'Ouest du 14 janvier 1909. En substance, cette chronique met en scène les courriers fictifs d'Yseult de Travannes adressés à son cousin Pierre qui vient de publier la correspondance de leur aïeule commune. Au fil des lettres d'Yseult, la déception première évolue en sentiment amoureux à l'égard de Pierre si bien que cette seconde correspondance finit par répéter la première.

\section{c) Le coin féminin : une certaine image de la France}

Présente en filigrane dans Contre le flot et de façon beaucoup plus visible dans Comme jadis, la France est omniprésente dans «Le coin féminin » dont Michelet a eu la charge pendant presque une dizaine d'années. Notre propos n'est pas ici de faire une recension systématique des mentions à la France dans les quelques quatre-cents chroniques publiées de façon hebdomadaire dans Le courrier de l'Ouest. Nous nous contenterons simplement d'en évoquer quelques grandes manifestations.

Référent culturel majeur du «Coin féminin », la France inspire à Michelet plusieurs recettes comme les «truites à la provençale », 1' «omelette aux huîtres » et les «carottes à la Bergesserin » (5 avril 1906) du nom de la commune en Saône-et-Loire d'où est originaire son père. Ce patriotisme gastronomique culminera avec la création le 20 septembre 1906 d'une section «Cuisine française» qu'inaugure la recette du pot-au-feu. Dans un registre moins anecdotique, Michelet choisit de publier dans sa chronique les poèmes d'un grand nombre d'auteurs français dont Paul Bourget, Jules Lemaitre Henri Lavedan, Louis Maigue, Rosemonde de Rostand et André Theuriet. Au fil des chroniques s'ajouteront à cette liste des noms d'auteurs canadiens-français comme Albert Lozeau dont les poèmes apparaissent pour la première fois dans la chronique 31 octobre 1907 et dont les textes reviendront régulièrement par la suite ${ }^{23}$. La rubrique compte également des poèmes d'auteurs franco-albertains comme Marie Boulanger, Églantine et Georges Ryval ${ }^{24}$. De même, Michelet publie de nombreux comptes rendus 
d'ouvrages canadiens-français comme Le secret de la marquise d'Adèle Bibaud (2 janvier 1908), Noëls anciens de la Nouvelle France d'Ernest Myrand (23 janvier 1908) et Une fleur mystique de la Nouvelle France de Marie-Catherine de Saint-Augustin (6 février 1908). Ainsi, dès la fin 1907, on assiste à une ouverture progressive à l'égard des lettres canadiennes-françaises dont Michelet devient une interlocutrice privilégiée avant d'en être une artisane à part entière. Par ailleurs, la rubrique «Le coin féminin » fait cohabiter des textes tirés de la presse canadiennefrançaise $^{25}$ (Album universel, Le journal de Françoise) et de la presse française (La maison rustique, La revue universelle).

Parmi les nombreux textes touchant de près ou de loin la France que compte la chronique féminine, certains s'attachent à laver la réputation de la femme française entachée par certaines croyances populaires vivaces quant à sa nature oisive et frivole («Femme française », 3 ; «Chronique » 3) : «Il est à désirer, pour l'union des deux rameaux de la même souche, que des Français traversent souvent l'Océan, pour venir loyalement, courageusement défendre une réputation que d'autres ont attaquée et attaquent encore [...] » (« Âme française », 3). D’autres textes à caractère autobiographique évoquent l'enfance française de Michelet, souvent à l'occasion de célébrations religieuses («Chandeleur », 3 ; «Souvenirs de la Sainte-Catherine », 3). Plusieurs articles tirés de revues françaises alimentent également les débats «locaux », en particulier sur la question du féminisme («Lettre de France » 3 ; « Article de Mgr Bolo », 5).

La question du vote des femmes âprement débattue en France, y compris dans les milieux catholiques, depuis le troisième Congrès féministe mondial de Paris en 1896 vient ainsi nourrir les discussions au sein de la francophonie canadienne («À travers la vie féminine », 3). Enfin, un petit nombre de chroniques datant des débuts du «Coin féminin » renseignent sur le positionnement idéologique de Michelet. C'est le cas en particulier d'un compte rendu que la chroniqueuse consacre à Angéline de Montbrun dans lequel elle revient sur l'opinion que Maurice se fait des Français :

Ce n'est pas au Havre, en débarquant, qu'on peut porter un jugement, et à Paris, quelle compagnie fréquente-t-il donc, qu'il ignorait ces phalanges laborieuses de jeunes gens catholiques?

N'était-il pas plutôt prévenu d'avance par la lecture des journaux canadiensfrançais qui, malencontreusement, puisent leurs opinions et leur inspiration dans des journaux français adversaires des catholiques ? Il ne manque cependant pas d'organes sérieux et indépendants, si l'on craint les exagérations de partis, où l'on trouverait la vérité vraie. 
Hélas ! Oui, il est une France ANTI-LIBERTAIRE, ANTI-RÉPUBLICAINE. » («Angéline de Montbrun », 3)

\section{Conclusion : exil ou renaissance?}

Fuyant sans doute la précarité et la politique anticléricale, les Michelet ont trouvé dans l'Ouest canadien une terre d'asile d'autant plus hospitalière qu'on y parlait français et que le catholicisme y avait encore droit de cité. Cet exil forcé s'est révélé être pour Marie Louise l'occasion d'une véritable renaissance. Sous le pseudonyme de Magali, elle connaît une ascension littéraire exceptionnelle qui la propulse du rôle de modeste chroniqueuse féminine à celui de porte-parole de la littérature canadienne-française. Fervente catholique, Michelet a très certainement retrouvé dans l'idéologie de l'Action française montréalaise un certain nombre de valeurs auxquelles sa famille (et son père, en particulier) était depuis longtemps attachée. En ce sens, son adhésion au discours clérico-nationaliste de l'abbé Groulx - avec qui elle a correspondu même après son retour en France - n'a rien de surprenant. Toutefois, on peut également se demander dans quelle mesure l'auteure de Comme jadis n'a pas également utilisé les moyens mis à disposition par l'Action française (Hébert, P.) pour lancer sa carrière littéraire et gagner une notoriété dont elle n'aurait pu rêver dans son coin d'Ouest. Le refus de publication qu'essuie sa troisième pièce «L'empreinte » en raison de son manque de caractère patriotique pourrait témoigner d'une prise de distance avec l'idéologie de l'action française découlant moins d'une prise de conscience critique que d'une volonté d'explorer de nouvelles thématiques. En ce sens, il nous semble que l'affiliation tenue pour acquise de Michelet au mouvement de l'abbé Groulx mériterait d'être davantage problématisée, ne particulier au regard des positions qu'elle exprime dans ses chroniques ${ }^{26}$. De même, il serait intéressant de comparer plus avant le type de féminisme revendiqué par la journaliste avec celui promu par des institutions comme la Fédération nationale de la Saint-Jean-Baptiste à la lumière des nuances qu'apportent des travaux comme celui de Karine Hébert en ce qui concerne la diversité de l'engagement social des femmes francophones. L'exactitude du portrait que Michelet brosse de l'anglomanie, lui ouvre à tout jamais les portes de l'institution littéraire canadienne-française au grand dam de ceux de ses frères d'armes qui stigmatisent ses origines françaises. Si les références à la France disparaissent presque totalement de Contre le flot, elles sont omniprésentes dans «Le coin féminin ». Ces références - notamment lorsqu'il s'agit d'articles prélevés dans des publications françaises viennent plutôt alimenter la discussion sur des sujets d'actualité nationaux ou provinciaux 
comme le vote des femmes. Plusieurs chroniques consacrées à la France s'attachent à corriger l'image négative souvent associée aux Français (et aux Françaises, en particulier). Aux nombreux articles consacrés à la France s'en ajoutent d'autres abordant des sujets plus locaux comme les méfaits de l'exode rural dans l'Ouest. Très rapidement, «Le coin féminin » montre une ouverture aux lettres canadiennes-françaises naissantes qu'elle cherche à promouvoir comme en témoignent les nombreux comptes rendus d'œuvres canadiennes-françaises. Située au carrefour de réseaux internationaux (France) et nationaux (Québec) d'information, «Le coin féminin » se présente comme un espace de socialité "transnationale» aussi diversifié que l'origine des lectrices auxquelles il donne la parole. Bien entendu, cette diversité demeure encadrée par un certain nombre de «convenances». Quant à Comme jadis, il reprend à son compte le format épistolaire de la chronique pour explorer sur un mode stéréoscopique les relations entre le Canada et la France. Dans ce contexte, le motif traditionnel de la conquête de l'Ouest n'est plus traité de façon linéaire et dramatique ; il se trouve inscrit dans la longue histoire des relations entre la France et le Canada, dont il représente une déclinaison. Loin de constituer une rupture historique ou géographique comme c'est le cas dans nombre de récits de pionniers, la Grande Guerre répète à la fois la guerre de la Conquête et le combat quotidien que livre le pionnier pour sa survie. Comme jadis est la célébration des valeurs communes qui président de part et d'autre de l'Atlantique aux succès de ces entreprises, à commencer par la vaillance et le patriotisme. En ce sens, le récit de la conquête de l'Ouest ne saurait être qu'exemplaire et édifiant.

\section{Bibliographie}

Bellerive, Georges. Nos auteurs dramatiques, anciens et contemporains, répertoire analytique. Québec : Garneau, 1933.

Bertin, Jacques. Du vent, Gatine! (Un rêve américain). Paris : Arléa, 1989.

Boivin Aurélien. «Louis Hémon et Maria Chapdelaine : deux centenaires à célébrer ». Cap-auxDiamants : la revue d'histoire du Québec 117 (2014) : 4-9.

«Chronique locale : l'émigration au Canada ». Le nouvelliste de l'Ouest. 12 décembre $1903: 2$.

Conan, Laure. Angéline de Montbrun. Montréal : Boréal, 2002.

Constantin-Bompard, Amélie. Au pays des érables. Tours : Maison Mame, 1934. 
Deligny, Louis (pseud. d'Olivier Maurault). «Comme jadis... Roman de Mlle Magali Michelet ». L'action française 1.10 (janvier 1926) : 53-56.

Dionne, Patrick. «Éclaircissements sur les prétendues mauvaises fréquentations littéraires de Lionel Groulx : le cas de Charles Maurras et de l'Action française de Paris ». Études d'histoire religieuse, 74 (2008) : 7-27.

Durieux, Marcel. Un héros malgré lui. Saint-Boniface : Plaines, 1986.

Eygun, Francois-Xavier. «Divergences et convergences à travers deux romans : La Pointe aux rats (1907) et L'aisance qui vient (1911) ». Actes du vingtième colloque du CEFCO 1518 octobre 2003 : L'Ouest : directions, dimensions et destinations. Winnipeg : PU de Saint-Boniface, 2005. 435-446.

Forestier, Georges. La Pointe-aux-Rats. Paris : Plon-Nourrit, 1907.

Frémont, Donatien. Sur le ranch de Constantin-Weyer. Winnipeg : Liberté, 1932.

Frénette, Yves, Marcel Martel et John Willis. Envoyer et recevoir. Lettres et correspondances dans les diasporas francophones. Laval : PU de Laval, 2006.

Gaulin, Michel. «Comme jadis..., roman de Magali Michelet». Dictionnaire des auvres littéraires du Québec, tome 2. Dir. Maurice Lemire. Montréal : Fides, 1980.

Harvey, Carol. J. «Souvenirs réels ou romancés ? Les écrits de Christine de La Salmonière et d'Amélie Constantin-Bompard ». Actes du vingtième colloque du CEFCO 15-18 octobre 2003 : L'Ouest : directions, dimensions et destinations. Winnipeg : PU de SaintBoniface, 2005. 447-461.

Hébert, Pierre. «Quand éditer, c’était agir : la bibliothèque de l'Action française (1918-1927) ». Revue d'histoire de l'Amérique française 46.2 (1992) : 219-244.

Hébert, Karine. «Une organisation maternaliste au Québec la Fédération nationale Saint-JeanBaptiste et la bataille pour le vote des femmes ». Revue d'histoire de l'Amérique française 52.3 (1999) : 315-344.

«La vie de l'action française ». L'action française 14.4 (octobre 1925) : 263.

Léo, Edmond [pseud. d'Armand Chossegros]. «Les livres. Comme jadis, par Magali Michelet ». Le devoir. 27 février $1926: 1$.

Maturié, Pierre. Athabasca terre de ma jeunesse. Edmonton : Institut pour le patrimoine de la francophonie de l'Ouest canadien. 
Michelet, Magali. Lettre à Lionel Groulx datée de 1955, Fonds Lionel Groulx, cote CLG1, série P1/A (correspondance), Banque Nationale des Archives du Québec.

---. Comme jadis. Paris : Petit écho de la mode, collection Stella, 1933.

---. « La vie de l'action française ». L'action française. 14.6 (décembre 1925) : 387-388.

---. Comme jadis. Montréal : Bibliothèque de l'Action française, 1925.

---. Contre le flot. Montréal : Bibliothèque de l'Action française, 1922.

---. Marraine de guerre. New York : MacMillan, 1922.

---. «Un article de Mgr Bolo ». Le courrier de l'Ouest. 4 mai 1911 : 5.

---. « Roman d'aïeule ». Le courrier de l'Ouest. 14 janvier 1909 : 2.

---. « Souvenirs de la Sainte-Catherine ». Le courrier de l'Ouest. 26 novembre 1908 : 3.

---. «L'âme française ». Le courrier de l'Ouest. 8 octobre 1908 : 3.

---. « La chandeleur ». Le courrier de l'Ouest. 30 janvier 1908 : 3.

---. « Chronique ». Le courrier de l'Ouest 18 avril $1907: 3$.

---. « À travers la vie féminine ». Le courrier de l'Ouest. 6 septembre $1906: 3$.

---. « La femme française ». Le courrier de l'Ouest. 23 août 1906 : 3.

---. « Lettre de France ». Le courrier de l'Ouest. 9 août $1906: 3$.

---. « Angéline de Montbrun ». Le courrier de l’Ouest. 28 juin 1906 : 3.

Morcos, Gamila. Dictionnaire des artistes et des auteurs francophones de l'Ouest canadien. Sainte-Foy : PU Laval, 1998.

« Notre concours d'art dramatique ». L'action française 14.5 (novembre 1925) : 63-64.

Painchaud, Robert. Un rêve français dans le peuplement de la Prairie. Saint-Boniface : Plaines, 1986.

«Première de Contre le flot au Gésu le 7 novembre ». La patrie. 4 novembre 1922 : 2.

Rao, Sathya. "'Le Coin féminin' de Magali : une rubrique féminine pas comme les autres », Cahiers d'études franco-canadiennes 25.1-2 (2013) : 129-153.

---. Entretien avec Mme Françoise Paretti fait à Agen (France) le 24 et 25 juin 2011 (non publié).

--- et Denis Lacroix. «Sur la piste de Magali Michelet : femme de lettres et chroniqueuse francophone de l'Ouest canadien ». Francophonies d'Amérique 34 (2012) : 173-190.

---. « Histoires de pionniers français dans l'Ouest canadien : le cas d'Un héros malgré lui de Marcel Durieux». Voix Plurielles 8.2 (2011): 65-78. 10 juin 2014. http://brock.scholarsportal.info/journals/voixplurielles/article/view/396/374 
Rousset, Jean. Forme et signification. Paris : Corti, 1962.

Roy, Max. «Contre le flot». Dictionnaire des ouvres littéraires du Québec, tome 2. Dir.

Maurice Lemire. Montréal : Fides, 1980 : 291-293.

Tessier, Jules. Américanité et francité. Essais critiques sur les littératures d'expression française en Amérique du Nord. Ottawa : Le Nordir, 2001.

Trofimenkoff, Susan Mann. Action française. French Canadian Nationalism in the Twenties. Toronto : U of Toronto P, 1975.

Viau, Robert. L'Ouest littéraire. Vision d'ici et d'ailleurs. Montréal : Méridien, 1992.

\section{Notes}

${ }^{1}$ Il n'est pas impossible que François Michelet ait assisté à une des conférences prononcées par l'abbé Gaire à Nantes. Celui-ci y vantait les mérites des plaines de l'Alberta où il avait déjà installé plusieurs Nantais (« Chronique locale $», 2$ ).

${ }^{2}$ Pour un portrait plus précis du statut socio-économique des Michelet, nous renvoyons le lecteur à notre article «Le Coin féminin' de Magali : une rubrique féminine pas comme les autres ».

${ }^{3}$ Comme le rappelle Robert Painchaud, l'accueil des immigrants d'origine française au Canada se heurtait à un certain nombre de préjugés de la part des autorités religieuses locales. Outre le fait que les Français passaient pour n'être pas de bons travailleurs, leur catholicisme altéré par la Révolution posait problème (168-169). En dépit de leur catholicisme, les Michelet sont des migrants atypiques dans la mesure où François est âgé de quarante-sept ans lorsqu'il s'embarque pour le Canada et ne possède, à notre connaissance, aucune compétence établie dans le domaine agricole ; or, ce sont principalement les jeunes agriculteurs que ciblent les missionnaires recruteurs. C'est dire que le succès de l'établissement de la famille au Canada reposait essentiellement sur les épaules de Charles Alexandre Michelet, cadet de la famille, âgé de dix-huit ans au moment du départ.

${ }^{4}$ Par Michelet nous désignons spécifiquement Marie Louise (ou Magali) qui constitue l'objet principal de cet article.

5 À notre connaissance, Michelet n'a rien publié après Comme jadis qui, s'il a été écrit en France et republié dans la collection française Stella, aborde les relations entre la France et le Canada ainsi que nous le verrons plus loin.

${ }^{6}$ Dans le cadre de cet article, nous ne lancerons pas dans la comparaison entre l'œuvre de Magali et celle de G. Bugnet qui, lui, n'est pas retourné en France.

${ }^{7}$ Nous n'incluons pas Marraine de guerre dans notre article car la pièce ne traite pas du Canada.

${ }^{8} \mathrm{Il}$ est fort probable que le roman ait été rédigé en partie ou intégralement à La Juynetière (Michelet, « La vie de l'action française », 387), lieu-dit près de la commune d'Étampes dans le département de l'Essonne. Le roman situe le château familial des Noulaine dans la localité d'Ormoy-la-rivière, également dans l'Essonne. À cela s'ajoute également plusieurs références précises à Étampes : le collège Geoffroy saint-Hilaire (81), l'église d'Ormoy (13), la vallée de la Juyne (13). Quant au château de Noulaine, il n'en existe pas de ce nom à Étampes ou dans ses environs. Peut-être s'agit-il d'une allusion déguisée au château de Goulaine en Loire-Atlantique où les Michelet avaient passé quelques années avant leur départ pour le Canada. Une partie de l'action de Comme jadis se déroule d'ailleurs dans la ville de Nantes.

${ }^{9}$ On notera cependant que Comme jadis n'est pas à strictement parler un roman épistolaire à deux voix. À la fin du roman, Herminie passe par Henri Maignan pour contacter Gérard. De plus, elle apprend le décès tragique de son bien-aimé de la plume de Marthe Leray.

${ }^{10}$ Il s'agit d'une coquille parmi les nombreuses que compte le roman.

${ }^{11}$ Cela dit, Comme jadis n'adopte pas le ton pessimiste d'un Georges Forestier dans La Pointe-aux-Rats (Eygun).

12 Publié en France en 1939, soit vingt ans après le séjour de l'auteure au Manitoba, Aux pays des érables s'apparente aux récits conventionnels de pionniers.

${ }^{13}$ Dans le contexte du début des années 1900, la notion de «féminisme » fait l'objet de nombreuses discussions au sein de la francophonie canadienne dont Michelet se fait l'écho dans ses chroniques. Critique à l'égard du féminisme politique des Anglo-saxonnes, elle revendique un engagement social conforme aux valeurs défendues par la Fédération nationale de la Saint-Jean-Baptiste. 
${ }^{14}$ Une telle connaissance ne privilégie pas le point de vue officiel des élites, mais plutôt celui d'une histoire « par en bas » dans une optique proche de celle de l'école des Annales.

${ }^{15}$ Maria Chapdelaine sera republié en France en 1921 dans la collection « Les cahiers verts » de Grasset.

${ }^{16}$ On peut penser que la parution dans cette collection féminine met au premier plan l'histoire d'amour entre Gérard et Herminie au détriment des aspects politiques et culturels du roman.

${ }^{17}$ Le nom de famille de l'écrivain français Gustave Aimard est mal orthographié par Magali.

${ }^{18}$ Toutefois, celle-ci aura l'occasion de racheter son égoïsme en s'enrôlant comme infirmière sur le front où elle se sacrifiera pour la France.

${ }^{19}$ Nous ne prenons pas ce terme dans son sens linguistique spécifique, mais dans un sens général que nous nous attacherons à préciser en contexte.

${ }^{20}$ Peut-être les Michelet appartenaient-ils eux-mêmes à ce contingent d'ouvriers.

${ }^{21}$ Comme montre Patrick Dionne, l'assimilation entre les positions de Maurras et de Groulx est loin d'aller de soi et demande à être problématisée. Chrétienne convaincue, il n'est pas certain que Michelet partage l'agnosticisme du maître de Martigues.

${ }^{22}$ Ce sacrifice semble abolir les barrières idéologiques entre les protagonistes français du roman, Henri, Jacqueline et Marthe.

${ }^{23}$ À titre d'exemple, treize de ses poèmes sont publiés dans la rubrique durant l'année 1908 qui a vu la publication de quarante-huit chroniques au total.

${ }^{24}$ Églantine et Georges Ryval sont tous les deux des pseudonymes.

${ }^{25}$ Magali compte un grand nombre de journalistes femmes de l'Est parmi ses correspondantes comme Georgine Lefaivre (alias Ginevra), Rose Monge (alias Rose de Provence) et Henriette Dessaulles (alias Jean Deshayes) (Rao, «Le coin féminin », 144-145).

${ }^{26}$ Nous n'avons pas abordé ce point dans le cadre restreint de cet article. 